\title{
ESTIMATION OF STRESS AND DISPLACEMENT INCREASE CAUSED BY HEAT GENERATION IN RUBBER VIBRATION ABSORBER
}

\author{
Svetlana Polukoshko ${ }^{1}$, Andris Martinovs ${ }^{2}$, Vladimirs Gonca ${ }^{3}$ \\ ${ }^{1}$ Ventspils University of Applied Sciences, Latvia; ${ }^{2}$ Rezekne Academy of Technologies, Latvia; \\ ${ }^{3}$ Riga Technical University, Latvia \\ pol.svet_a@inbox.lv, andris.martinovs@ru.lv, vladimirs.gonca@rtu.lv
}

\begin{abstract}
Rubber and rubber-like materials (elastomers) are widely used for anti-vibration mounts and shock absorbers for vehicles, machinery, building structures due to their specific properties: ability to absorb vibration and shock loads, low elastic modulus, high mechanical strength, high elongation at brake, reversible elastic deformation. Rubber is a material that is capable of recovering from large deformations quickly and forcibly, which is suitable for work under cyclic loading. During deformation elastomeric materials absorb in an irreversible way part of the energy, causing this deformation. The energy absorbed during each cycle heats the deformed rubber element and dissipates in media. Heat generation in rubber causes additional stresses and deformations which are poorly known, and they are a subject of our study. In the presented paper the work of a rubber anti-vibration mount in the form of a straight circular cylinder under action of cyclic loading is studied. Poisson's ratio of the rubber material is $\mu=0.5$, the weight of the mount is not taken into account. Temperature field is assumed known based on the previous work (it depends on the frequency and amplitude of vibration, heat conductivity and heat capacity of the material, etc.). The stress-strain state analysis was carried out based on the Reissner variational principle. Analytical dependences for temperature additions to stresses and displacements are derived that allows estimating stiffness of the anti-vibration mount and its increase as a result of self-heating. Obtained results may be useful for proper design of anti-vibration mounts allowing changing geometrical dimensions in order to reach the required temperature field.
\end{abstract}

Keywords: rubber vibro-absorber, cyclic loading, heat generation, variational methods, additional stress.

\section{Introduction}

Elastomeric materials, like natural or synthetic rubber, have unique physical-and-mechanical properties: high elasticity, ability to absorb vibration and shock loads, high mechanical strength, high resistance to abrasion, reversible elastic deformation, low volume compressibility, linear relationship between stress and strain up to strain of $15-20 \%$. Due to these evident engineering advantages elastomeric materials are widely used for shock and vibration absorbers for vehicles, machinery and buildings $[1 ; 2]$.

The unique mechanical properties of elastomers make them suitable for applications in which cyclic loads are involved. Due to the high hysteresis losses present in such materials, significant heat generation is observed. This effect, combined with low thermal conductivity, leads to a temperature increase in rubber parts, which is usually called heat storage. Large dynamic deformations appearing during the operation of the elastic elements of vibration insulators cause significant thermal fields. A change in temperature compared to the initial one leads to a change in volume, and, consequently, to the appearance of additional stresses.

The question of determining the temperature fields of self-heating is widely covered in the literature, but the question of estimating the magnitude of temperature stresses and deformations is poorly known, it is usually mentioned that these stresses are small [3-8].

In given paper the work of a rubber vibration absorber of cylindrical form with preliminary compression under cyclic loading is investigated. The work of this absorber is considered in the region of small deformation (15-20\%), i.e. in zone of linear relationship between stress and strain.

For the region of small deformations in engineering calculations some assumptions are used to analyze the temperature fields of self-heating. In particular, it is assumed that the influence of thermal stresses and strains on the temperature of self-heating may be neglected; weight of rubber is not taken into account. The elastomeric materials are nearly volumetrically incompressible, i.e. their Poisson ratio $\mu$ is extremely close to 0.5 , for example natural rubber $\mu \sim 0.4900-0.4995$, since in given work for further calculation it is assumed that $\mu=0.5$.

For the purpose of calculating heat generation problems in cylindrical and prismatic bodies it is convenient to use direct variational methods: principle of minimum of total potential energy [8-10], principle of minimum of the additional potential energy of deformation $[8 ; 11]$ and the Reissner 
variational principle [9;12]. The formulation of the variation principle depends on the functions characterizing the state of the deformed body. In the principle of minimum of total potential energy there are displacement functions, in the principle of minimum of the additional potential energy stresses functions, in the Reisner's variational principle - functions of displacements and stresses. These functions are subject to variation (infinitely small changes) in order to obtain the variation equation. All other functions are considered to be related to the main varying functions by physical dependencies (Hooke's law etc.).

In given work the Reissner variational principle is used; variational equation $\Delta \Phi=0$ (where $\Phi$ Reissner functional) is equivalent to a system of six relationships between the components of the stress tensor and the strain tensor, three equilibrium equations and boundary conditions.

Elastic stresses and displacements, caused by force, are approximated by the functions satisfying the boundary conditions and compatibility equations. Substituting approximating functions to the Reissner's functional for elastic case and solving the variational equations, we receive the constants of approximating functions and all parameters of rubber work may be found. Temperature additions to stress and displacements are also approximated by corresponding functions and these functions are substituted to the Reissner's functional for the heat conduction problem.

The stiffness of the vibration isolator and the relative stiffness increase are determined depending on the self-heating temperature, geometrical dimensions and mechanical-and-physical properties of the elastomer. Physical and mechanical properties are considered to be constant during operating because the temperature rising is not large.

Symbolic and numerical calculations are fulfilled using Mathcad 15 program - suitable software for engineering design. The obtained results allow engineers to determine more accurately the size of vibroabsorbers.

\section{Materials and methods}

In this research the work of the elastomeric anti-vibration mount in the form of a solid straight circular cylinder is considered. The scheme of the anti-vibration mount is shown in Fig. 1: the elastomeric cylinder is reinforced by two rigid metal plates, which is considered non-deformable; the plates are fixed to both ends of the elastomeric cylinder by gluing.

a)

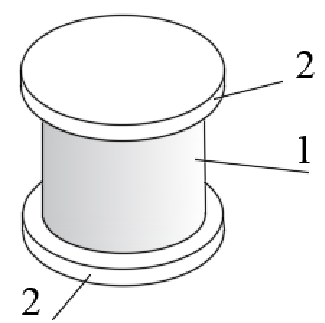

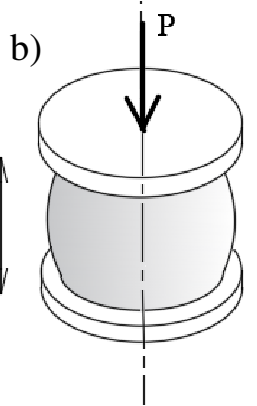
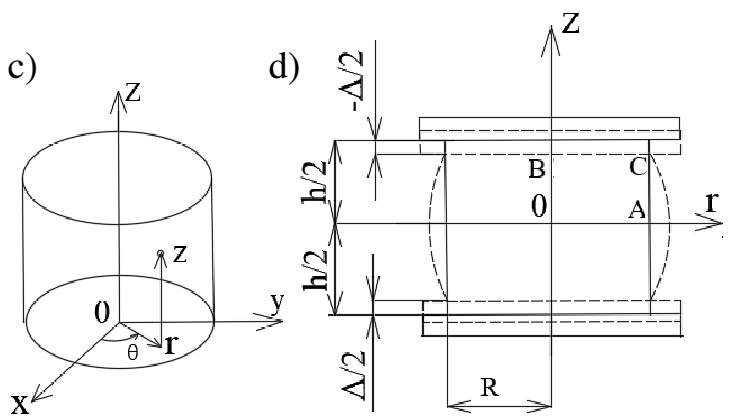

Fig. 1. Investigated object: a - cylindrical form vibro-absorber ( 1 - elastomeric cylinder; 2 - rigid metal plate); $b$ - deformation of vibro-absorber under action of axial force or kinematic axial excitation and temperature; $\mathrm{c}$ - cylindrical coordinate system; $\mathrm{d}$ - design scheme of absorber

Cylindrical coordinate system $0 r \theta z$ is applied for further calculation, $\Delta-z$-displacement of the cylinder.

Vibro-isolation mount is preliminary compressed on $\Delta$ value. The rubber element under consideration is a body of rotation, to which axially symmetrical loads are applied. Since each meridional plane $z 0 r$ is a plane of symmetry, there cannot be tangential stresses in the meridional planes. In the planes, which are perpendicular to $z$ axis, tangential stresses are parallel to the meridional plane.

Stresses components to be determined $\sigma_{z}, \sigma_{\theta}, \sigma_{r}, \sigma_{r z}$ are shown in Fig. 2. These stresses must be considered as functions of $z$ and $r$. 


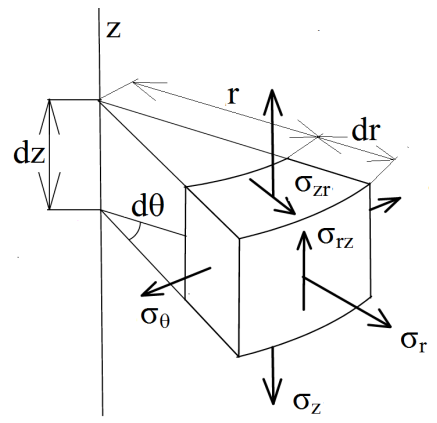

Fig. 2. Scheme of stresses in axially symmetrical cylinder

For determining the functions characterizing the stress-strain state of the deformed body it is convenient to use direct variational methods. The problem of finding these functions is reduced to a definite integral of these functions (composed function or the functional), and the functions themselves are found from the condition of the extremum of this functional. We set the displacement functions, satisfying the boundary conditions and compatibility equations, as well as the stress functions depending on the coordinates and on unknown coefficients. Then we substitute these functions into the equation of the functional and calculate a definite integral as a function of unknown coefficients. Equating to zero the variation of the resulting expression, we determine the unknown coefficients. Substituting these coefficients to the stresses and displacement functions, we obtain the sought-for functions. In our study the solution is implemented using the Reissner variational principle, the corresponding functional for the case of an incompressible material $(\mu=0.5)$ in a cylindrical coordinate system has the form [7; 9]:

$$
\begin{gathered}
\Phi=\int_{V}\left\{-\frac{1}{4 G}\left[\left(\sigma_{r}^{e}\right)^{2}+\left(\sigma_{\theta}^{e}\right)^{2}+\left(\sigma_{z}^{e}\right)^{2}\right.\right. \\
\left.+2\left(\sigma_{r z}^{e}\right)^{2}-\frac{1}{3}\left(\sigma_{r}^{e}+\sigma_{z}^{e}+\sigma_{\theta}^{e}\right)^{2}\right]+u_{r, r}^{e} \sigma_{r}^{e}+\frac{u_{r}^{e}}{r} \sigma_{\theta}^{e}+\mu_{z, z}^{e} \sigma_{z}^{e}+ \\
\left.+\left(u_{r, z}^{e}+u_{z, r}^{e}\right) \sigma_{r z}^{e}\right\} d V,
\end{gathered}
$$

where $G$-shear modulus of elastomer, $\mathrm{kPa}$;

$u_{i}$ - displacement function components the $i$-axis $(r, \theta, z), \mathrm{cm}$;

$u_{i, j}$ - first derivation of $u_{i}$ function on $j$;

$\sigma_{i}$ - stress components along the $i$-axis $(r, \theta, z), \mathrm{kPa}$;

$V$ - volume of rubber cylindrical vibroisolator, $\mathrm{cm}^{3}$.

Upper index $e$ in notation means elastic. Geometrical boundary condition for displacement:

$$
u_{r}^{e}\left(r, z= \pm \frac{h}{2}\right)=0, u_{z}^{e}\left(r, z= \pm \frac{h}{2}\right)=\mp \frac{\Delta^{e}}{2}, \frac{\partial u_{r}^{e}}{\partial z}\left(z= \pm \frac{h}{2}\right) \neq 0, \frac{\partial u_{r}^{e}}{\partial z}(z=0)=0 .
$$

For studying case we have one compatibility equation:

$$
\frac{\partial^{2} \varepsilon_{r}}{\partial z^{2}}+\frac{\partial^{2} \varepsilon_{z}}{\partial r^{2}}=2 \frac{\partial^{2} \varepsilon_{r z}}{\partial z \partial r}
$$

Elastic displacement functions, satisfying boundary conditions (2) and compatibility equation (3) are accepted as:

$$
u_{r}^{e}(r, z)=A^{e} r\left(1-4 \frac{z^{2}}{h^{2}}\right), u_{z}^{e}(r, z)=-\frac{3 \Delta^{e}}{2 h} z\left(1-\frac{4}{3}{\frac{z^{2}}{h^{2}}}^{2}\right)
$$

Elastic stress functions are set:

$$
\sigma_{z}^{e}=C_{1}^{e}+C_{2}^{e} r^{2}+C_{3}^{e} z^{2}, \sigma_{r}^{e}=\sigma_{\theta}^{e}=B_{1}^{e}\left(R^{2}-r^{2}\right), \sigma_{r z}{ }^{e}=B_{2}{ }^{e} r z\left(R^{2}-r^{2}\right) .
$$

By substituting displacements and stresses functions (4) and (5) into (1) and volume integration, we receive functional $\Phi$ as a function of constants $A^{e}, \Delta^{e}, C_{1}{ }^{e}, C_{2}{ }^{e}, C_{3}{ }^{e}, B_{1}{ }^{e}, B_{2}{ }^{e}$. From stationary conditions of functional $\Phi$ we receive the constants: 


$$
A^{e}=\frac{3 \Delta}{4 h}, C_{1}^{e}=-\frac{G \Delta}{2 h}\left(4 \rho^{2}+9\right), C_{2}^{e}=\frac{2 G \Delta}{h^{3}}, C_{3}^{e}=\frac{18 G \Delta}{h^{3}}, B_{1}^{e}=-\frac{2 G \Delta}{h^{3}}, B_{2}^{e}=-\frac{12 G \Delta}{R^{2} h^{3}} .
$$

Substituting eqs. (6) into eqs. (4), (5), we determine displacement and stress functions for elastic case. Then $\Delta^{e}-P^{e}$ dependence of the cylindrical anti-vibration mount is obtained:

$$
P^{e}=\pi R \Delta^{e} G \rho\left(\rho^{2}+3.6\right) \text {, or } \Delta^{e}=\frac{P^{e} h}{\pi R^{2} G} \frac{1}{\left(\rho^{2}+3.6\right)} \text {, where } \rho=\frac{R}{h} .
$$

In the case of solving the heat conduction problem, the functional for determining the temperature additions to displacements and stresses has the form [7;9]:

$$
\begin{gathered}
\Phi^{a}=\int_{V}\left\{-\frac{1}{4 G}\left[\left(\sigma_{r}^{a}\right)^{2}+\left(\sigma_{\theta}^{a}\right)^{2}+\left(\sigma_{z}^{a}\right)^{2}+2\left(\sigma_{r z}^{a}\right)^{2}-\frac{1}{3}\left(\sigma_{r}^{a}+\sigma_{\theta}^{a}+\sigma_{z}^{a}\right)^{2}\right]+u_{r, r}^{a} \sigma_{r}^{a}+\frac{u_{r}^{a}}{r} \sigma_{\theta}^{a}+u_{z, z}^{a} \sigma_{z}^{a}+\right. \\
\left.+\left(u_{r, z}^{a}+u_{z, r}^{a}\right) \sigma_{r z}^{e}-\alpha \tilde{T}\left(\sigma_{r}^{a}+\sigma_{\theta}^{a}+\sigma_{z}^{a}\right)\right\} d V,
\end{gathered}
$$

where $\tilde{T}-$ temperature field in the elastomeric cylinder, which is above environment, deg $\mathrm{C}$; $\alpha$ - heat conductivity factor, $\operatorname{deg} \mathrm{C}^{-1}$.

Upper index $a$ in notation means addition. The temperature that arises in a rubber cylinder depends on the frequency of vibration, the heat transfer coefficient and the coefficient of heat deposition. Temperatures distribution field in the rubber cylinder is taken according to equation [9; 13]:

$$
\tilde{T}(r, z)=C_{T}\left(r^{2}-\zeta\right) \cdot\left(z^{2}-\beta\right), \text { where: } \zeta=\frac{2 R}{H_{1}}+R^{2}, \beta=\frac{h}{H_{2}}+\frac{h^{2}}{4},
$$

where $H_{1}$ - relative heat changing factor from rubber to media, $\mathrm{cm}^{-1}$;

$\mathrm{H}_{2}$ - relative heat changing factor from rubber to metal, $\mathrm{cm}^{-1}$;

$C_{T}$ - heat capacity factor, deg $\cdot \mathrm{cm}^{-4}$.

$C_{T}$ depends on the frequency of vibration $\omega\left(\mathrm{s}^{-1}\right)$, heat transfer factor $\lambda$, absorption coefficient $\eta$, indicating which part of energy is absorbed per cycle $[7 ; 9 ; 13]$.

This choice of equation (9) ensures the fulfillment of boundary conditions on the free surface:

$$
\tilde{T}_{, r}+H_{1} \tilde{T}=0 \text {, if } r=R, \tilde{T}_{, z}+H_{2} \tilde{T}=0 \text {, if } z= \pm h / 2 .
$$

Geometrical boundary condition for this case:

$$
u_{r}^{a}\left(r, z= \pm \frac{h}{2}\right)=0, u_{z}^{a}\left(r, z= \pm \frac{h}{2}\right)=\mp \frac{\Delta^{a}}{2}, \frac{\partial u_{r}{ }^{a}}{\partial z}\left(z= \pm \frac{h}{2}\right) \neq 0, \frac{\partial u_{r}{ }^{a}}{\partial z}(z=0)=0 .
$$

Elastic displacements functions, satisfying boundary conditions and compatibility equation are set:

$$
u_{r}^{a}(r, z)=A^{a} r\left(1-4 \frac{z^{2}}{h^{2}}\right), u_{z}^{a}(r, z)=\frac{3 \Delta^{a}}{2 h} z\left(1-\frac{4}{3}{\frac{z^{2}}{h^{2}}}^{2}\right) .
$$

Elastic stress functions are accepted:

$$
\sigma_{z}^{a}=C_{1}{ }^{a}+C_{2}{ }^{a} r^{2}+C_{3}{ }^{a} z^{2}, \sigma_{r}{ }^{a}=\sigma_{\theta}{ }^{a}=B_{1}{ }^{a}\left(R^{2}-r^{2}\right),{\sigma_{r z}}^{a}=B_{2}{ }^{a} r z\left(R^{2}-r^{2}\right) \text {. }
$$

Stresses functions (13), displacement functions (12) and the temperature field function (9) are substituted into (8) and after volume integration we receive functional $\Phi^{a}$ as a function of constants $A^{a}$, $a^{e}, C_{a}^{e}, C_{2}{ }^{a}, C_{a}{ }^{e}, B_{a}^{e}, B_{a}{ }^{e}$. From stationary conditions of functional $\Phi^{a}$ we receive the constants:

$$
A^{a}=-\frac{3 \Delta^{a}}{4 h}+\frac{9 C_{T} \alpha}{4} \zeta_{1} \beta_{1}, \text { where: } \zeta_{1}=\zeta-\frac{R^{2}}{3}=\frac{2 R}{H_{1}}+\frac{2 R^{2}}{3}, \beta_{1}=\beta-\frac{h^{2}}{12}=\frac{h}{H_{2}}+\frac{h^{2}}{6},
$$




$$
\begin{gathered}
C_{1}^{a}=\frac{G \Delta^{a}}{2 h}\left(4 \rho^{2}+9\right)-\frac{G C_{T} \alpha}{8}\left(48 \rho^{2} \zeta_{1} \beta_{1}-R^{2} h^{2}+24 \zeta \beta\right), \\
C_{2}^{a}=-\frac{2 G \Delta^{a}}{h^{3}}-\frac{6 G C_{T} \alpha}{h^{2}}\left(\frac{h^{2}}{2}+\zeta_{1}\right) \beta_{1}, C_{3}^{a}=-\frac{18 G \Delta^{a}}{h^{3}}+3 G C_{T} \alpha \zeta_{1}, \\
B_{1}^{a}=\frac{2 G \Delta^{a}}{h^{3}}-\frac{6 G C_{T} \alpha}{h^{2}} \zeta_{1} \beta_{1}, B_{2}^{a}=\frac{12 G \Delta^{a}}{R^{2} h^{3}}-\frac{36 G C_{T} \alpha}{R^{2} h^{2}} \zeta_{1} \beta_{1} .
\end{gathered}
$$

Then, additional force $P^{a}$, acting in $z$-direction and $z$-direction displacement $\Delta^{a}$ is determined:

$$
\begin{gathered}
P^{a}=3 \pi R^{2} G C_{T} \alpha\left(\rho^{2} \zeta_{1} \beta_{1}+\zeta_{2} \beta_{2}\right), \zeta_{2}=\zeta-\frac{R^{2}}{2}=\frac{2 R}{H_{1}}+\frac{R^{2}}{2}, \beta_{2}=\beta-\frac{h^{2}}{20}=\frac{h}{H_{2}}+\frac{h^{2}}{5}, \\
\Delta^{a}=\frac{3 h C_{T} \alpha}{\rho^{2}+3.6}\left(\rho^{2} \zeta_{1} \beta_{1}+\zeta_{2} \beta_{2}\right), \text { or } \Delta^{a}=\frac{P^{a} h}{G \pi R^{2}\left(\rho^{2}+3.6\right)} .
\end{gathered}
$$

The relation between $P^{a}$ and $P^{e}$ is received:

$$
k=\frac{P^{a}}{P^{e}}=3 h C_{T} \alpha \frac{\left(\rho^{2} \zeta_{1} \beta_{1}+\zeta_{2} \beta_{2}\right)}{\Delta^{e}\left(\rho^{2}+3.6\right)} .
$$

It is assumed in eq. (5) that $\sigma_{z}^{e}$, like $\sigma_{z}^{a}$, is a function of $r$ and $z$ axis only for comparing $P^{a}$ and $P^{e}$ forces and obtaining eq. (16). In fact, the elastic $z$-axial stress is uniformly distributed along the $z$ axis. Accounting it and also complicating $\sigma_{r}^{e}$, supposing its dependence on $z$, we obtain the constants for elastic stress definition. For $\sigma_{r z}{ }^{e}$ the stress function and constant remained the same as (5) and (6).

$$
\begin{gathered}
\sigma_{z}^{e}=C_{1}^{e}+C_{2}{ }^{e} r^{2}, \sigma_{r}^{e}=\sigma_{\theta}{ }^{e}=B_{1}{ }^{e}\left(R^{2}-r^{2}\right)+B_{3}{ }^{e} z^{2}\left(R^{2}-r^{2}\right), \\
C_{1}^{e}=-\frac{G \Delta^{e}}{h}\left(2 \rho^{2}+3.9\right), C_{2}{ }^{e}=\frac{G \Delta^{e}}{h}\left(2 \rho^{2}+0.9\right), B_{1}{ }^{e}=-\frac{2 G \Delta}{h^{3}}, B_{3}{ }^{e}=\frac{G \Delta}{R^{2} h^{3}}(2 R-1.35 h) .
\end{gathered}
$$

For the final conclusion what function of stress is better, it is necessary to carry out the experimental testing of such damper with different dimensions. It will be the next stage of our work.

\section{Results and discussion}

The results of numerical solution of stresses and displacements for the damper, shown in Fig, 1, in accordance with above equations are given below for the anti-vibration mount with dimensions: radius $R=2.5 \mathrm{~cm}$, height $h=4 \mathrm{~cm}, \Delta^{\mathrm{e}}=0.1 \mathrm{~cm}$ and physical-and-mechanical characteristics: $G=3 \mathrm{MPa}$, $H_{1}=0.4 \mathrm{~cm}^{-1}, H_{2}=52.40 \mathrm{~cm}^{-1}, \alpha=10^{-4} \mathrm{deg}^{-1}, C_{T=} 0.5425 \mathrm{deg} \cdot \mathrm{cm}^{-4}$. Data are taken form $[7 ; 9 ; 13]$.

Fig. 3 shows the elastic $z$-axial stress, calculated according to eqs, (17). In Fig. 4 the additional temperature to ambient one, arising inside the absorber, is presented. In Fig. 5 a) relative stiffness $P^{a} / P^{e}$ increase depending on the radius is shown for $h=4 \mathrm{~cm}$, in Fig. $5 \mathrm{~b}$ ) relative stiffness $P^{a} / P^{e}$ depending on $h$ for $R=2.5 \mathrm{~cm}$ in accordance with formula (16).

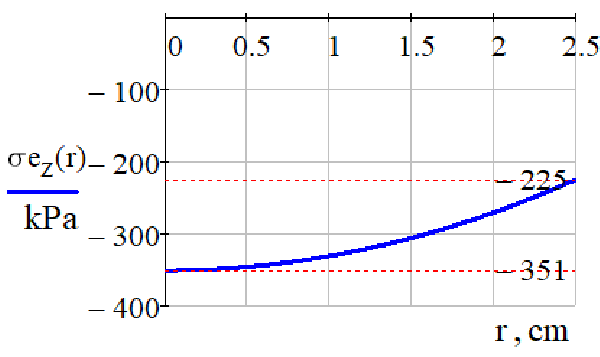

Fig. 3. Elastic axial stress $\sigma_{z}{ }^{e}=f(r)$ acording to eqs. (17)
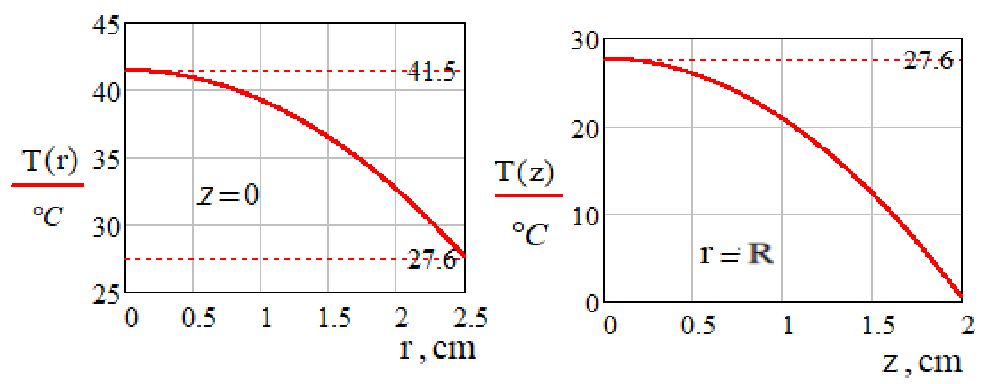

Fig. 4. Temperature increase inside rubber cylinder depending on $r$ (left) and depending on $z$ (right) 
a) $P^{a} / P^{e}=f(r)$

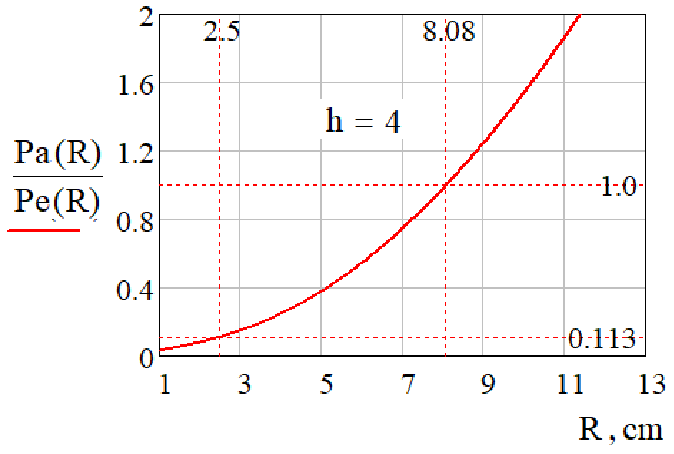

b) $P^{a} / P^{e}=f(z)$

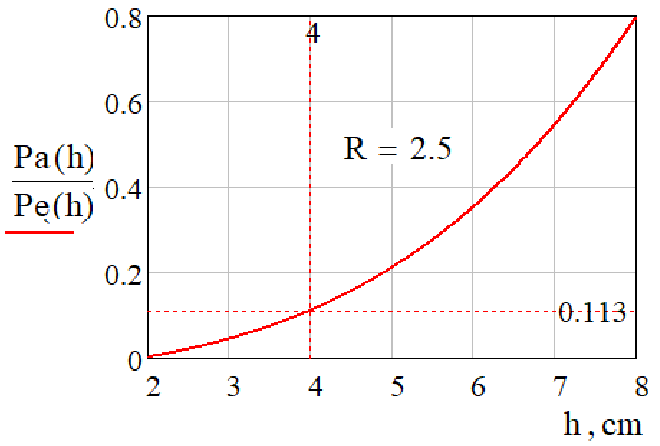

Fig. 5. $\boldsymbol{P}^{a} / \boldsymbol{P}^{e}$ ratio for cylindrical absorber: a - depending on radius $R$, if $h=4 \mathrm{~cm}$; $\mathrm{b}$ - depending on height $h$, if $R=2.5 \mathrm{~cm}$

In Fig. 6-10 the plots of dependence of $\sigma_{z}{ }^{e}, \sigma_{z}{ }^{a}, \sigma_{r}{ }^{e}, \sigma_{r}^{a}$ and $\sigma_{r z}{ }^{e}, \sigma_{r z}{ }^{a}$ stresses on coordinate are presented, elastic stresses are defined according to eqs. (5), (6). In Fig. 7, 9 the temperature additions $\sigma_{z}{ }^{a}$ are shown more detailed. In Fig. 11 the displacements of the rubber cylinder in $r$-and $z$-direction are shown.
a) $\sigma_{z}=f(r)$
b) $\sigma_{z}=f(r)$

$\mathrm{r}, \mathrm{cm}$
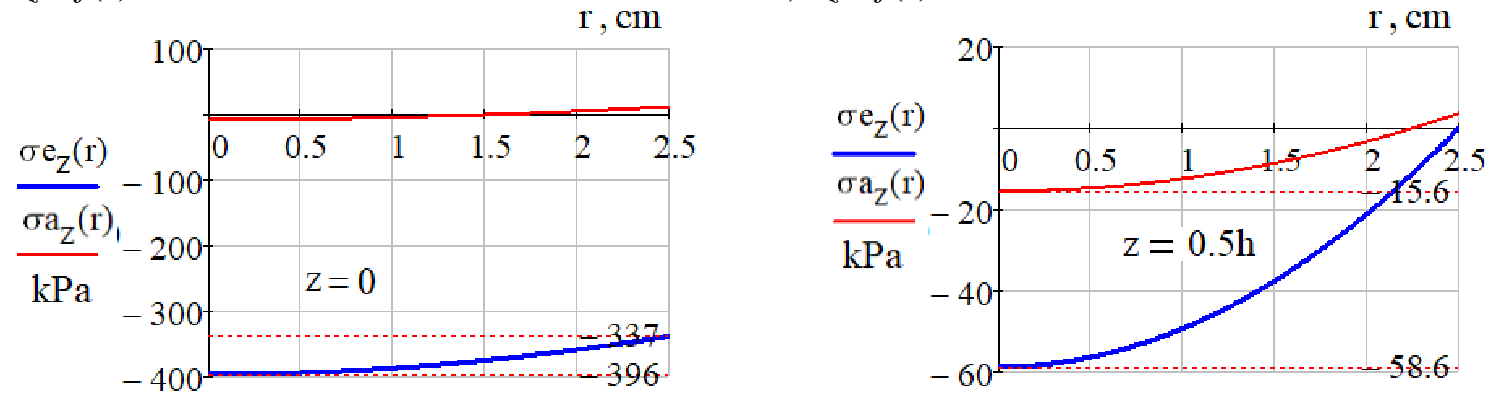

Fig. 6. Elastic compression stress $\sigma_{z}{ }^{e}$ and temperature additions $\sigma_{z}{ }^{a}$ dependences on $\boldsymbol{r}$ : $\mathrm{a}-\sigma_{z}=f(r)$ in the middle plane; $\mathrm{b}-\sigma_{z}=f(r)$ in the upper and lower plane

a) $\sigma_{z}{ }^{a}=f(r)$

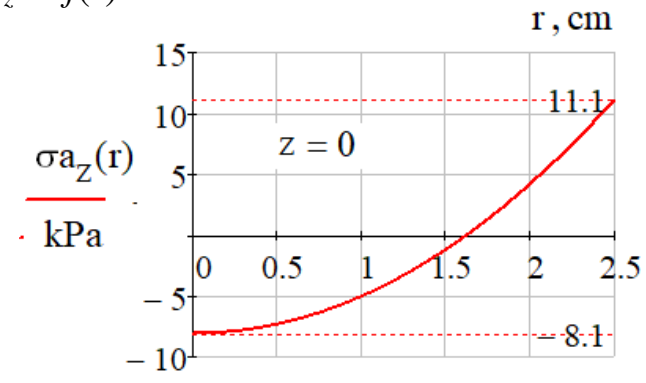

b) $\sigma_{z}^{a}=f(r)$

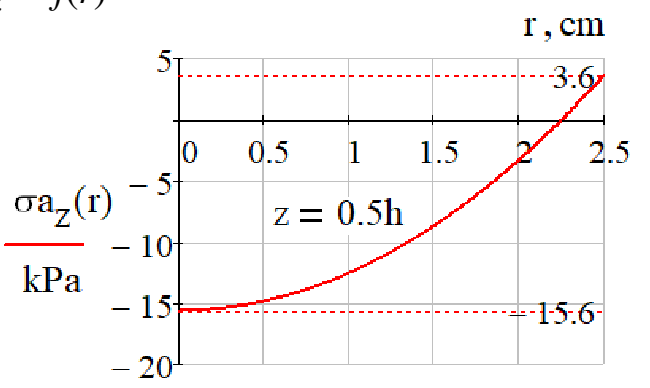

Fig. 7. Temperature additions $\sigma_{z}{ }^{a}$ dependences on $r:$ a $-\sigma_{z}^{a}=f(r)$ in the middle plane; $\mathrm{b}-\sigma_{z}{ }^{a}=f(r)$ in the upper and lower planes

a) $\sigma_{z}=f(z)$

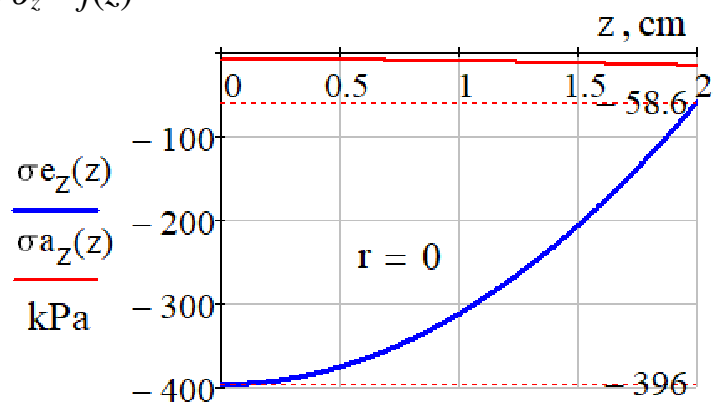

b) $\sigma_{z}=f(z)$

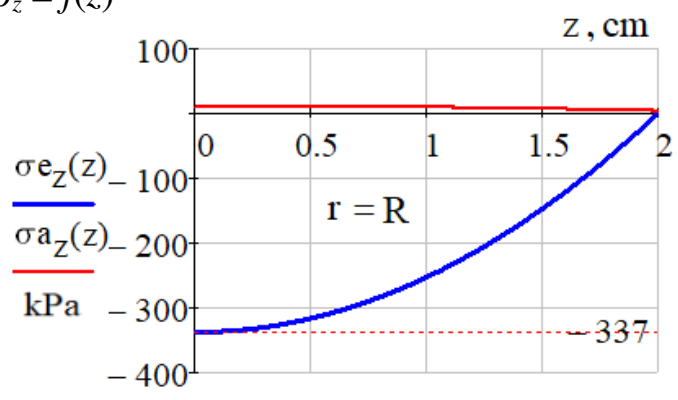

Fig. 8. Elastic compression stress $\sigma_{z}{ }^{\mathrm{e}}$ and temperature additions $\sigma_{\mathrm{z}}{ }^{\mathrm{a}}$ dependences on $z$ : $\mathrm{a}-\sigma_{z}=f(z)$ for $r=0 ; \mathrm{b}-\sigma_{z}=f(z)$ for $r=R$ 
a) $\sigma_{z}^{a}=f(z)$

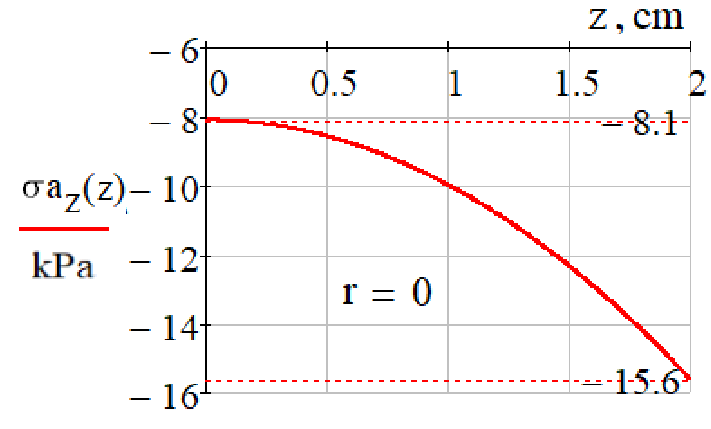

b) $\sigma_{z}^{a}=f(z)$

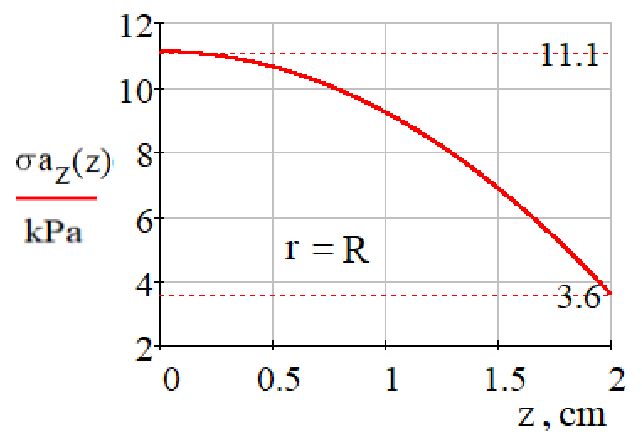

Fig. 9. Temperature additions $\sigma_{z}{ }^{a}$ dependences on $z: \mathrm{a}-\sigma_{z}{ }^{a}=f(z)$ if $r=0 ; \mathrm{b}-\sigma_{z}{ }^{a}=f(z)$ if $r=R$

a) $\sigma_{r=f(r)}$

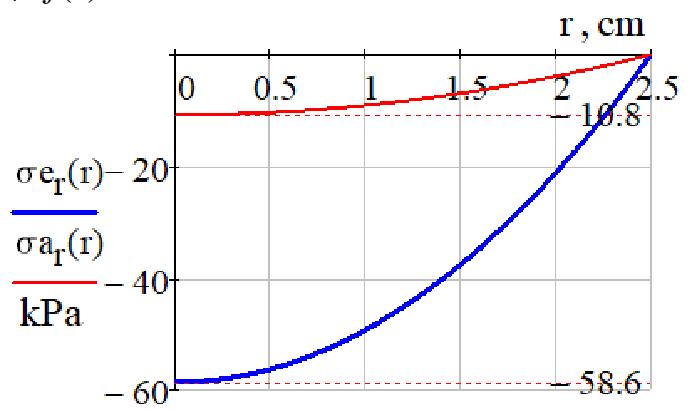

b) $\sigma_{r z}=f(r)$

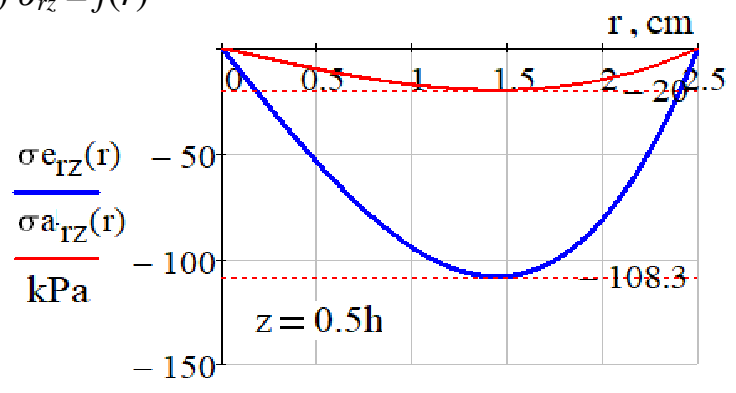

Fig. 10. Elastic stress and temperature additions dependences on $r$ : a $-\sigma_{r}{ }^{e}=f(r), \sigma_{r}{ }^{a}=f(r)$ for any $z, \mathrm{~b}-{\sigma_{r z}}^{e}=f(r),{\sigma_{r z}}^{a}=f(r)$ for upper and lower planes of rubber cylinder

a) $u_{r=f} f(z)$

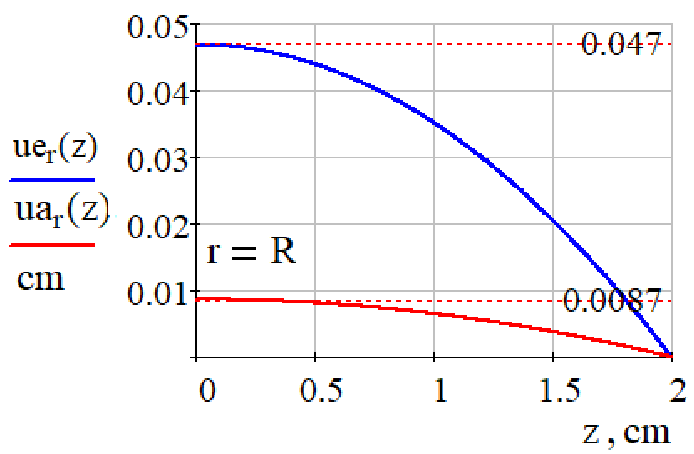

b) $u_{z}=f(z)$

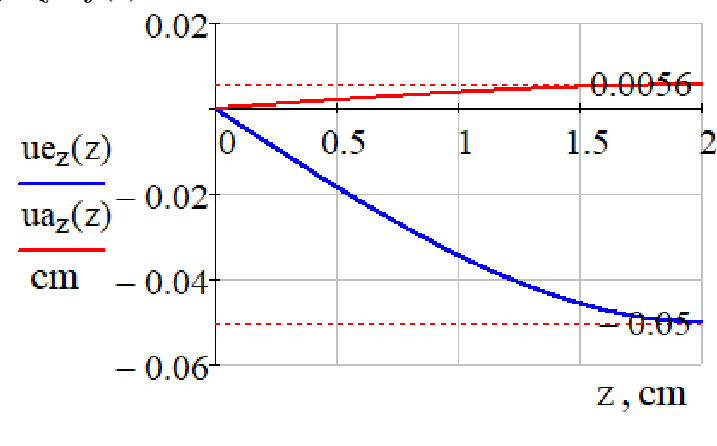

Fig. 11. Displacements of cylindrical absorber as results of force action and as results of temperature increase: $\mathrm{a}-u_{r=} f(z)$ when $r=R ; \mathrm{b}-u_{z}=f(z)$

Values of stresses and displacements in characteristic points, indicated in Fig. 1.d), calculated in accordance with the aforementioned equations are presented in Table 1. Here the elastic stress $\sigma_{z}^{e}$ calculated according to eqs. (5), (6) is given to the left on line and according to eqs. (17) to the right on line.

Table 1

Stress and displacement values in characteristic points

\begin{tabular}{|c|c|c|c|c|c|c|c|c|}
\hline $\begin{array}{c}\text { Stresses and } \\
\text { displacements } \\
\text { in point }\end{array}$ & $\begin{array}{c}\boldsymbol{\sigma}_{z}{ }^{e}, \\
\mathbf{k P a}\end{array}$ & $\begin{array}{c}\boldsymbol{\sigma}_{z}{ }^{a}, \\
\mathbf{k P a}\end{array}$ & $\begin{array}{c}\boldsymbol{\sigma}_{\boldsymbol{r}}{ }^{\boldsymbol{e}}, \\
\mathbf{k P a}\end{array}$ & $\begin{array}{c}\boldsymbol{\sigma}_{\boldsymbol{r}}{ }^{\boldsymbol{}}, \\
\mathbf{k P a}\end{array}$ & $\begin{array}{c}\boldsymbol{u}_{z}{ }^{e}, \\
\mathbf{m m}\end{array}$ & $\begin{array}{c}\boldsymbol{u}_{z}{ }^{a}, \\
\mathbf{m m}\end{array}$ & $\begin{array}{c}\boldsymbol{u}_{\boldsymbol{r}}{ }^{e}, \\
\mathbf{m m}\end{array}$ & $\begin{array}{c}\boldsymbol{u}_{\boldsymbol{r}}{ }^{a}, \\
\mathbf{m m}\end{array}$ \\
\hline 0 & $-396 /-351$ & -8.1 & -58.6 & -10.8 & 0 & 0 & 0 & 0 \\
\hline $\mathrm{A}$ & $-338 /-225$ & 11.1 & 0 & 0 & 0 & 0 & 0.47 & 0.087 \\
\hline $\mathrm{B}$ & $-59 /-351$ & -15.6 & -58.6 & -10.8 & -0.5 & 0.056 & 0 & 0 \\
\hline $\mathrm{C}$ & $0 /-225$ & 3.6 & 0 & 0 & -0.5 & 0.056 & 0 & 0 \\
\hline
\end{tabular}


Let us assume that temperature is the same inside the rubber cylnder $35^{\circ} \mathrm{C}$. Substituting the constant value $T$ to eqs.(7) instead of the temperature field $\widetilde{T}(z, r)$ and using the same procedure, we define that the displacements increase in 1.6 and stress approximately in 2 times.

\section{Conclusions}

1. The calculation of the stress and deformation appearing in the rubber cylindrical vibroabsorber as the result of temperature increase is presented in given work. Calculation was performed applying the Reissner variational principle with help of Mathcad 15 software.

2. The solution shows that the increase of temperature gives small addition to the stresses and displacements and can be neglected, but in some kind of elastomers may lead to crack and fatigue and must be carefully controlled by selecting proper dimensions of the vibroisolator.

3. For the preliminary compressed anti-vibration mount subsequent heating of the vibration isolator can significantly change its rigidity, since it depends on the ratio of the geometric dimensions that change with heating. Many engineering installations, like vibration screens, sifting machines, sieving units, are loaded in this order (with preliminary compression and subsequent vibratory loading), therefore the design of anti-vibration mounts for these machines requires special care and verification according to the proposed method.

\section{References}

[1] Gent A.N. Engineering with Rubber: How to Design Rubber Components. Munich: Carl Hanser Verlag, 2011. $428 \mathrm{p}$.

[2] Bauman J. T. Fatigue, Stress and Strain of Rubber Components: Guide for Design Engineers. Carl Hanser Verlag, Munich, 2008. 214 p.

[3] Terazaki K., Okada M. Heat generation and thermal conduction in rubber with cyclic compression, Heat Transfer - Ja panese Research, 7 (1978)1, pp. 40-60.

[4] Banič M. S., Stamenkovic D. S., Miltenovic V. D. etc. Prediction of Heat Generation in Rubber or Rubber-Metal Springs. THERMAL SCIENCE, Vol. 16, Suppl.2, 2012, pp. S527-S539.

[5] Schieppati J., B. Schrittesser B., Wondracek A.etc. Heat build-up of rubbers during cyclic loading. Proceedings of the 11th European Conference on Constitutive Models for Rubbers (ECCRM XI), June 2019, Nantes, France. Eds Bertrand Huneau, etc. Taylor \& Francis, London (2019) [online][11.02.2020] Available at: https://www.researchgate.net/publication/334680030

[6] Le Chenadec J., Kaoult I., Stolz C. etc. Cyclic approximation of the heat equation in finite strains for the heat build-up problem of rubber. Journal of Mechanics of Materials and Structures, 2009, Vol. 4, No. 2, 2009, pp. 309-318.

[7] Потураев В.Н., Дырда В.И. Резиновые детали машин (Rubber Machine Elements). Moscow: Mashinostrojenie, 1977. 217 p. (In Russian).

[8] Лавендел Э.Э. Расчет резинотехнических изделий (Design of fabricated rubber products). Moscow: Mashinostroenie, 1976. 182 p. (In Russian).

[9] Дымников С.И., Лавендел Э.Э., Павловскис А-М.А еtс. Прикладные методы расчёта изделий из высокоэластичных материалов. (Applied methods of calculating the product of highly elastic materials). Rīga: Zinātne, 1980. 238 p. (In Russian).

[10] Polukoshko S., Gonca V., Martinovs A., etc. Boundary conditions influence on compressive stiffness of elastomeric isolators. Proceedings of the 15-th Engineering for rural development, 25.27.05.2016, Jelgava, Latvia. pp. 737-744.

[11] Polukoshko S., Gonca V. Calculation methods and fields of use of multilayered rubber-metal packages. In Constitutive Models for Rubber IX. (Marvalova B., Petrikova, I., Eds.), Proceeding of ECCMR IX, Prague, 2015, pp. 623-630.

[12] Reissner E. On a variational theorem in elasticity. Journal of Mathematics and Physics 1950. Vol. 29, N 2, pp. 90-95.

[13] Ляпунов В.Т., Лавендел Э.Э., Шляпочников С.А. Резиновые виброизоляторы, справочник. (Rubber vibration isolators, handbook). Leningrad: Sudostrojenie, 1988. 216 p. (In Russian). 